\title{
Improving door to needle times with nurse initiated thrombolysis
}

\author{
P Wilmshurst, A Purchase, C Webb, C Jowett, T Quinn
}

\begin{abstract}
Objective-To evaluate the effect of nurse initiated thrombolysis on door to needle time (the interval between arriving at the hospital and starting thrombolytic treatment) in patients with acute myocardial infarction.

Design-Comparison of door to needle times before and after the employment of nurses trained and approved to initiate thrombolysis without prescription by a doctor but with a protocol for rapid triage of patients with chest pain.

Setting-A district general hospital.

Subjects-All patients admitted with suspected myocardial infarction between April 1995 and March 1999.

Main outcome measures-Speed (door to needle time) and appropriateness of administration of thrombolytic drugs to patients with acute myocardial infarction who gave a characteristic history and had appropriate criteria on the admission ECG.

Results-During seven periods (each of four months) before the introduction of nurse initiated thrombolysis and a new chest pain triage protocol, the median door to needle time varied from 50-58 minutes. In four periods (each of 4-6 months) following the introduction of the changes, the median door to needle time was 25-30 minutes. The improvement was significant $(\mathrm{p}<0.001)$. Nurses trained to initiate thrombolysis currently provide cover for $66 \%$ of the time. Median door to needle time for nurses was 15 minutes. Median door to needle time for junior doctors improved to 35 minutes. The median door to needle times when nurses initiated thrombolysis was significantly shorter than when doctors did so $(p<0.001)$. There have been no inappropriate management decisions by nurses approved to initiate thrombolysis.

Conclusions-The use of nurse initiated thrombolysis has resulted in a clinically important reduction in the time taken for thrombolysis to be started in patients with acute myocardial infarction.
\end{abstract}

(Heart 2000;84:262-266)

Keywords: thrombolysis; acute myocardial infarction; door to needle time

Mortality from myocardial infarction is reduced by rapid reperfusion of infarct related arteries by the timely administration of thrombolytic drugs. The greatest improvements in survival are in those patients treated most quickly. ${ }^{1}$ Delays in administration of thrombolytic drugs may occur before a patient is admitted to hospital, particularly in rural areas where patients may be remote from the nearest ambulance base and district hospital. When symptoms are atypical or the initial ECG is non-diagnostic there may be delays in hospital in initiation of thrombolysis, but in those cases the benefit to risk ratio of thrombolytic treatment is less than in patients with unequivocal myocardial infarction on admission. ${ }^{1}$ In many cases of unequivocal infarction on admission (defined in this study as typical symptoms with onset of pain less than 12 hours earlier and an admission ECG showing unequivocal ST elevation or left bundle branch block which was not known to be old), the interval between arrival at hospital and the initiation of thrombolytic drug treatment, known as the door to needle time, is considerably greater than the nationally proposed standard of 30 minutes. $^{2}$

The Royal Shrewsbury Hospital admits 250-300 patients each year in whom the primary discharge diagnosis is myocardial infarction. Approximately three quarters of those with a primary discharge diagnosis of acute myocardial infarction receive thrombolytic treatment, and approximately three quarters have an unequivocal myocardial infarct on admission. In April 1995, the hospital became part of the West Midlands Region audit of treatment of myocardial infarction. This report describes the methods used to analyse our door to needle times and to get these within the proposed 30 minute standard.

\section{Methods}

During seven consecutive four month audit periods between April 1995 and July 1997 the total number of patients admitted to this hospital with a primary discharge diagnosis of acute myocardial infarction was 625 . Of these, 437 were given thrombolytic treatment and 463 had an unequivocal myocardial infarct on admission. The audit returns showed a median door to needle time for unequivocal myocardial infarct on admission varying from $50-58$ minutes (fig 1). The reasons for delay in initiation of thrombolytic treatment were analysed in 40 consecutive patients with unequivocal myocardial infarction on admission and in whom door to needle time was greater than 30 minutes. Fifty two reasons for delays were identified in the 40 patients. The delays could be divided into three categories: 


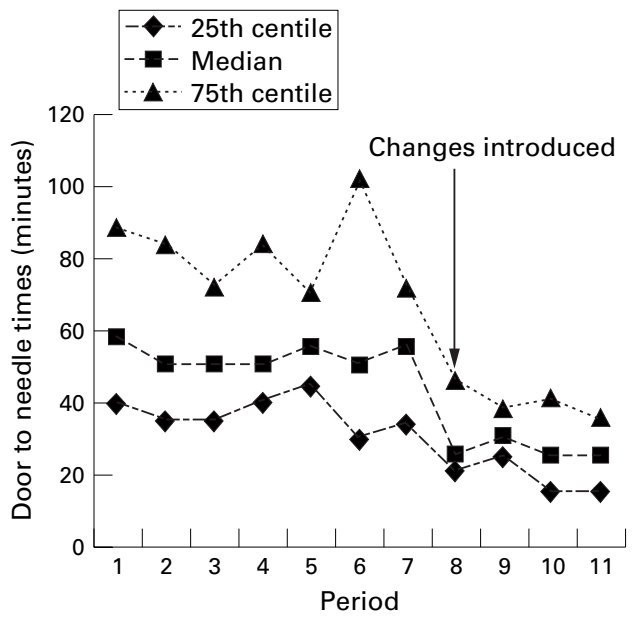

Figure 1 Door to needle times for cases of unequivocal myocardial infarct on admission during the seven audit periods before and the four audit periods after introduction of nurse initiated thrombolysis and a chest pain triage trolley.

(1) Forty two per cent of delays were because the patients were not transferred to the coronary care unit rapidly from their point of entry to the hospital. Some patients were admitted directly to the coronary care unit, but many were admitted through the accident and emergency department or the medical admissions ward and a few went through both. Those not admitted directly to the coronary care unit did not receive thrombolytic drugs until transferred to the unit. In many cases delayed transfer to the coronary care unit was because there was no bed available on the unit. In other cases, junior doctors admitted the patients through the admission ward for their own convenience, because this enabled them to clerk all admissions in one place.

(2) Thirty five per cent of delays were because junior doctors were unable to attend and assess the patient speedily. Despite regular teaching sessions and introduction of guidelines in which the necessity of speed in initiating thrombolytic drugs was emphasised, there was no significant improvement in the time taken for junior doctors to attend. Doctors failed to respond speedily even when "fast bleeped".

(3) Twenty three per cent of delays occurred in cases seen speedily by a junior doctor (house physician or senior house officer) but the decision was deferred to a more senior doctor, usually the medical registrar. In addition, when reviewed subsequently there were cases where decisions by junior doctors to give or withhold thrombolytic drugs were considered inappropriate by a consultant cardiologist.

The possibility of thrombolysis at the point of admission of patients to the hospital was considered impractical because of the lack of specialist nurses outside the coronary care unit. The hospital is on a split site across a main road and medical admissions arrive at more than one unit. Staff in other areas were unwilling to train to give thrombolytic drugs, and providing extra cardiac trained nurses in other areas would have been expensive. We therefore made two major changes to the protocol for managing patients with suspected myocardial infarction.

First, we arranged with the ambulance service, local doctors, and the accident and emergency department that patients with chest pain would be admitted directly to the coronary care unit for assessment and, when appropriate, initiation of thrombolysis on a cardiac triage trolley. The trolley was placed in a space from which one bed had been removed. Even though this removed one bed from the eight beds in the unit we assessed that it would result in less blockage of beds on the unit. A trolley could not be used to house a patient for longer than necessary for assessment and initiation of treatment. Patients with noncardiac pain were discharged home or transferred to a general medical ward, as appropriate. Patients with cardiac pain were transferred to a bed in the unit and if all the other seven beds on the unit were full the fittest patient on the unit was transferred to the adjacent cardiac stepdown ward. If necessary patients on the cardiac ward were moved elsewhere to make a bed available.

Second, when a patient arrived on the coronary care unit the admitting junior doctor was fast bleeped to attend. Meanwhile coronary care nurses inserted a venous cannula, performed a 12 lead ECG, and started the assessment of the patient using a proforma checklist (see appendix). One of the nurses, who had undertaken special training and assessment, made the decision about whether to give thrombolytic treatment if no doctor had arrived by the time the nurse assessment was completed. The nurse's decision was made without discussion with or authorisation by a doctor. If the decision was to give thrombolytic treatment the infusion was started immediately.

The eventual aim was to provide 24 hour cover on the coronary care unit by nurses approved to initiate thrombolysis. Initially four coronary care nurses (and later a fifth) went through a training and assessment package. The nurses selected each had a coronary care certificate and at least five years of experience working in coronary care. The main aim of the teaching package was to train the nurses in protocol guided interpretation of the history, basic physical signs, and ECG to confirm the diagnosis of unequivocal myocardial infarction on admission, and to exclude other causes of chest pain (such as aortic dissection and pericarditis) and contraindications to thrombolysis. The teaching package also included revision of cannulation and advanced life support skills. The choice of thrombolytic agent was laid out in the protocol; accelerated recombinant tissue plasminogen activator to be given to patients with anterior myocardial infarcts, to patients who had been given streptokinase four or more days previously, and to hypotensive patients. Streptokinase to be used in other patients requiring thrombolysis. Following an examination of competence based on vignettes as described by Quinn and 
colleagues, ${ }^{3}$ the nurses were accredited to initiate thrombolysis.

Before instituting the protocol there was an "intention to treat month," during which thrombolysis nurses assessed patients with chest pain and suspected myocardial infarction. The nurses recorded whether or not they would have instituted thrombolysis and the time at which the decision was made. The nurse's decision and the time taken to reach it were compared with the management decision made by the junior doctor who saw the patient and the actual door to needle time. During this "intention to treat month" 27 patients were assessed by thrombolysis nurses. The nurses made decisions more rapidly than junior doctors in all cases. The nurses' decisions agreed with those of junior doctors on 23 occasions, and were considered to be correct decisions when they were reviewed by a consultant cardiologist. In four patients the decisions of nurses were more conservative than those of the doctors. The nurses would have withheld thrombolytic drugs in four patients who were later prescribed them by a junior doctor.

Two of the audit periods since introduction of these changes were also of four months' duration, but two (periods 10 and 11) were changed to six months' duration for purely administrative reasons after cessation of the West Midlands Region audit of treatment of myocardial infarction. During the 20 months that comprised these four audit periods between August 1997 and March 1999, the total number of patients admitted to this hospital with a primary discharge diagnosis of acute myocardial infarction was 477 . Of these, 308 were given thrombolytic treatment and 336 had unequivocal myocardial infarction on admission.

\section{Results}

Figure 1 shows the 25th, 50th, and 75th centiles for door to needle times in patients with unequivocal myocardial infarction on admission. Nurse initiated thrombolysis was instituted after period 7 . The median door to needle time for unequivocal myocardial infarcts was reduced from 50-58 minutes before implementation of these changes to 25-30 minutes afterwards $(\mathrm{p}<0.001)$.

Currently five thrombolysis nurses provide $66 \%$ cover for the unit (but we hope to train three more thrombolysis nurses to give 24 hour cover). Many patients receive thrombolysis at times when there was no thrombolysis nurse on duty. Figure 2 shows a comparison of the 25 th, 50 th, and 75 th centiles for door to needle times in patients with unequivocal myocardial infarction on admission when thrombolytic treatment was initiated by a thrombolysis nurse and by a junior doctor, during the three month period October to December 1998. The door to needle times in patients whose thrombolytic treatment was initiation by thrombolysis nurses were shorter than when treatment was initiated by junior doctors $(\mathrm{p}<0.001)$. However, these groups are not entirely comparable. Specifically, doctors were on duty for the whole 24

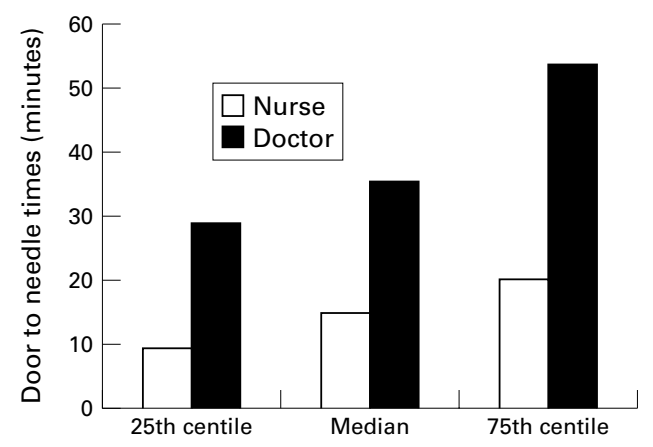

Figure 2 Comparison of door to needle times for nurse and doctor initiated thrombolysis for cases of unequivocal myocardial infarct on admission during the period October to December 1998.

hour period but thrombolysis nurses were not, and nurses had the option to leave a decision to be made by a doctor when they are in doubt about the correct action. The shortest door to needle time for a thrombolysis nurse was seven minutes. The median door to needle time for junior doctors was reduced compared with that before the introduction of our changes (from 50-58 to 35 minutes).

We identified no case in which a nurse has given a thrombolytic drug to a patient who did not have protocol criteria of unequivocal myocardial infarction or in whom the protocol excluded thrombolytic treatment. In addition, assessment by nurses has led to earlier recognition of previously unsuspected medical conditions (aortic dissection and pericarditis). There has also been an improvement in the way myocardial infarcts are managed by junior doctors. They now attend patients on the coronary care unit more quickly and use the same proforma as the nurses to guide treatment. This has resulted in less inappropriate medical treatment. Whether these changes in behaviour of junior doctors are because they recognise the dissatisfaction with their previous performance, or because of a competitive attempt to initiate treatment, we cannot judge.

The use of the triage trolley has increased the number of patients passing through the coronary care unit by $51 \%$. The great majority of these patients have chest pain which is not the result of either myocardial infarction or unstable angina, and these patients are rapidly transferred elsewhere. This increased throughput has required an increase in numbers of coronary care unit staff.

\section{Discussion}

Many hospitals have implemented mechanisms to improve door to needle times. "Fast track" treatment of patients has been shown by others to reduce median door to needle times from 93 minutes to 49 minutes. ${ }^{4}$ Though this is a dramatic reduction in the median time, it is still well outside the nationally proposed standard of 30 minutes and it was similar to the median time at the Royal Shrewsbury Hospital before the introduction of our current protocol. In the study by Pell and colleagues, fast tracking took place at the point of first contact in the accident and emergency department. ${ }^{4}$ Our first point of 
contact is now the coronary care unit and we have ensured rapid access to it.

The main innovation in this study was the extension of the role of coronary care nurses to assess patients with chest pain and give thrombolytic drugs without prescription by a doctor. It has been shown in intention to treat studies that appropriately trained nurses can reliably assess patients with suspected myocardial infarction. ${ }^{35}$ Caunt reported the first initiative in which specialist nurses gave patients with myocardial infarction thrombolytic treatment without the patient being assessed by a doctor. ${ }^{6}$ She reported preliminary data in 24 patients given thrombolysis by a specialist nurse and 45 for whom the drugs were prescribed by junior doctors. ${ }^{6}$ The door to needle times for patients treated by the specialist nurse were dramatically shorter.

In this study we report the effect of introduction of the type of specialist nurse reported in the small study by Caunt on routine care of patients with acute myocardial infarction in the coronary care unit of a large district general hospital. It is difficult to draw conclusions from comparisons before and after introduction of a change in management because factors which influence outcomes may also alter during the same time. In this case the introduction of thrombolysis nurses was also accompanied by changes in admission protocols for patients with chest pain. From a scientific point of view it would have been better to make a single change in management and to randomise patients to pre-existing and new managements. In our district hospital with limited resources such a scientific study was not practical. Our primary aim was to complete the audit loop by identifying the problems, and apply solutions to improve patient care-in this case by shortening door to needle times without reduction in safety. However, the dramatic and sustained reduction in door to needle times immediately nurse initiated thrombolysis was introduced, and the comparison between nurse thrombolytic times and junior doctor thrombolytic times, strongly suggest that nurse initiated thrombolysis was the major factor in reducing door to needle times.

Other data also point to benefit to patients from these changes in to the management of acute myocardial infarction. A recent National Health Service (NHS) executive analysis of "clinical indicators" showed that the introduction of new protocols for the management of acute myocardial infarction at the Royal Shrewsbury Hospital corresponded with a significant as well as clinically important $(65 \%)$ reduction in 30 day mortality for myocardial infarction. ${ }^{7}$ In 1995-96 the average 30 day mortality for myocardial infarction at the Royal Shrewsbury Hospital was $14532 / 100000$ (95\% confidence interval (CI) 9897 to 19 166). In $1997-98$ the mortality was $5388 /$ 100000 (95\% CI 2823 to 7953). According to these statistics, in 1997-98 the 30 day mortality for myocardial infarction at the Royal Shrewsbury Hospital was the second lowest in the
NHS and the lowest in the hospital category (large acute hospital). It is not possible to show that the improved 30 day mortality for myocardial infarction at our hospital was directly attributable to the introduction of nurse initiated thrombolysis, but the coincidence of these events is suggestive.

Our changes were tailored to the problems we identified by clinical audit. Different solutions may be appropriate in other hospitals. Somauroo and colleagues have reported the use of a different type of thrombolysis nurse. ${ }^{8}$ In their study one nurse provided 37 hours of cover in the accident and emergency unit to triage patients with chest pain and identify those admitted with unequivocal myocardial infarction and thereby expedite a doctor's prescription of a thrombolytic drug. Their thrombolysis nurse was not authorised to give thrombolytic drugs without explicit instruction in each case. ${ }^{9}$ Even with only 37 hours covered each week, these investigators showed that such a nurse could dramatically improve door to needle time.

The introduction of thrombolysis initiated by nurses using a protocol for rapid assessment of patients with chest pain at the Royal Shrewsbury Hospital has appreciably reduced in-hospital delays in initiating thrombolysis without reduction in safety. We have demonstrated a safe and effective way of extending the role of specialist nurses to improve care for patients with suspected acute myocardial infarction.

We thank Christina Harris, Rosalind Stanley, and Marian Davies, who are thrombolysis nurses and sisters on the coronary care unit, and Sian Michelmore and Nicola Jones who helped with data collection. We also thank the staff of the coronary care unit, the audit department, and the medical care group unit, the audit department, and the medical care group
management team at the Royal Shrewsbury Hospital for their assistance. All the authors were involved in the discussions from which this project evolved. CW wrote the first draft of the prowhich this project evolved. CW wrote the first draft of the protocol and all authors helped in revising the protocol. AP and CW supervised collection of data. CJ analysed the data. TQ and PW assessed the nurses before "licensing" them to act as thrombolysis nurses. PW wrote the first draft of the paper. All authors amended drafts. PW accepted clinical responsibility for the actions of thrombolysis nurses and guarantees the paper.

1 Fibrinolytic Therapy Trialists' Collaborative Group. Indications for fibrinolytic therapy in suspected acute myocardial infarction: collaborative overview of early mortality and major morbidity results from all randomised trials of more than 1000 patients. Lancet 1994;343:311-22

2 De Bono DP, Hopkins A, on behalf of the Joint Audit Committee of the British Cardiac Society and the Royal College of Physicians. The management of acute myocardial infarction: guidelines and audit standards. $\mathcal{F} R$ Coll Physicians Lond 1994;28:312-17.

3 Quinn T, MacDermott A, Caunt J. Determining patients' suitability for thrombolysis: coronary care nurses' agree-
ment with an expert cardiological "gold standard" as assessed by clinical and electrocardiographic "vignettes." Intensive Crit Care Nurs 1998;14:219-24.

4 Pell ACH, Miller HC, Robertson CE, et al. Effect of "fast track" admission for acute myocardial infarction on delay track" admission for acute myocardial
to thrombolysis. BMf 1992;304:83-7.

5 Quinn T. Can nurses safely assess suitability for thrombolytic therapy? A pilot study. Intensive Crit Care Nurs 1995;11:126-9.

6 Caunt J. The advanced nurse practitioner in CCU. Care Crit Ill 1996;12:136-9.

7 NHS Executive. Quality and performance in the NHS: clinical indicators. London: Department of Health, 1999.

8 Somauroo JD, McCarten P, Appleton B, et al. Effectiveness of a "thrombolysis nurse" in shortening delay to thrombolysis in acute myocardial infarction. $\mathcal{F} R$ Coll Physicians Lond 1999;33:46-50.

9 Wilmshurst P. What is meant by "thrombolysis nurse"? [letter] f R Coll Physicians Lond 1999;33:284-5. 
CORONARY CARE UNIT

Nurse Initiated Thrombolysis

PATIENT ASSESSMENT FORM

\begin{tabular}{|l|l|l|}
\hline Patient Name: & \multicolumn{2}{|l|}{ P.I.D: } \\
\hline Patient Address: & DOB: & \multicolumn{2}{|l|}{ Age: } \\
\cline { 2 - 3 } & Cons: & Time: \\
\cline { 2 - 3 } affix patient label here & D.O.A. & \\
\hline
\end{tabular}

\section{Admitting Diagnosis:}

Suggested by:

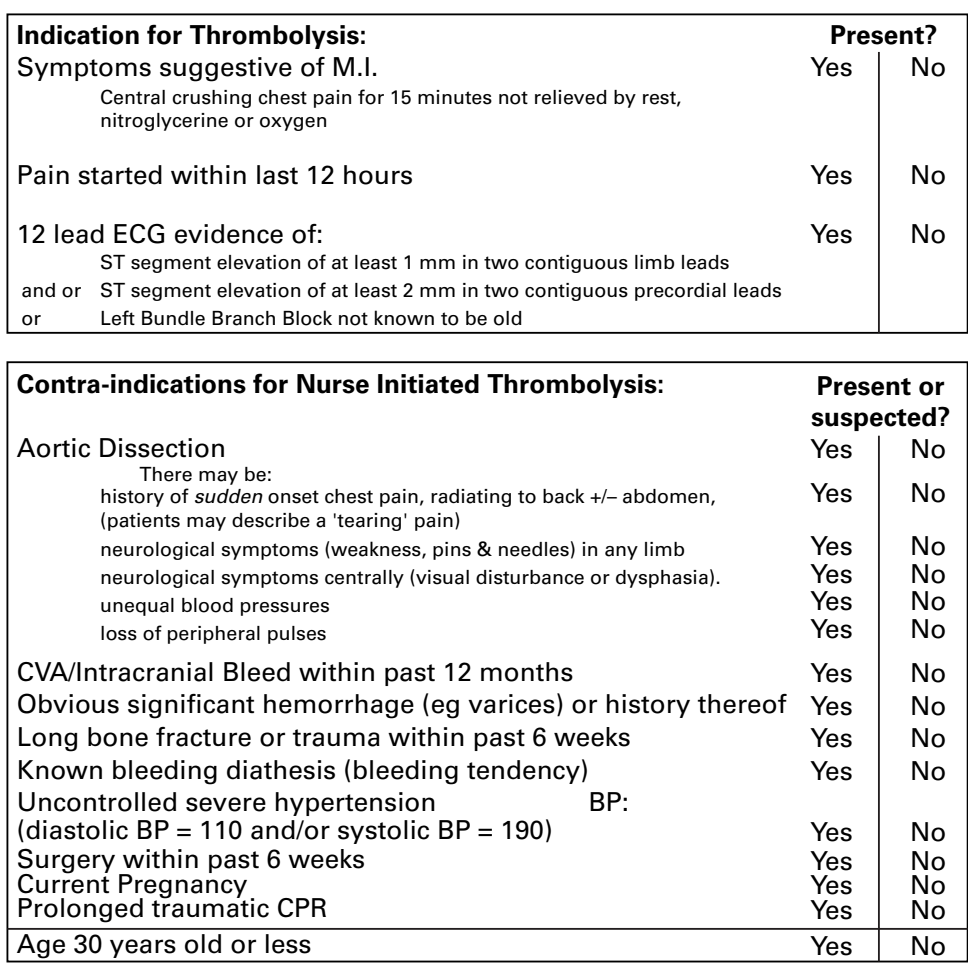

\begin{tabular}{|cc|c|}
\hline $\begin{array}{c}\text { Assessment/Action Plan } \\
\text { Are there clear indications for thrombolysis? }\end{array}$ & Yes & No \\
If yes, are there any identified or suspected contra-indications? & Yes & No \\
If no, time nurse initiated thrombolysis commenced: & Time: \\
$\quad$ time doctor attended patient & Time: \\
If yes, time doctor 'fast bleeped' & Time: \\
time doctor attended patient & Time: \\
\hline
\end{tabular}

Thrombolytic Agent Chosen:

Rationale:

Time thrombolysis commenced:

Door to needle time (minutes):

\begin{tabular}{|l|l|l|}
\hline Signature: & Name: & Date: \\
\hline
\end{tabular}

